\title{
Heat Transfer in the Steady Flow of a Second-Order Fluid between Two Enclosed Discs Rotating With Different Angular Velocities
}

\author{
K.R. SINGH \& SHIVA \\ Reader,Department of Mathematics, Meerut College, Meerut-250001 (U.P.) India \\ Research Scholar,Department of Mathematics, Meerut College, Meerut-250001 (U.P.) India
}

\begin{abstract}
The problem of heat transfer in the steady flow of an incompressible second-order fluid between two enclosed discs rotating with different angular velocities has been discussed. The effect of elastico-viscosity, cross-viscosity parameters and ratio of the angular velocity on the temperature profile and Nusselt number for the cases of radial outflow and inflow have been investigated in the regions of no-recirculation and recirculation.
\end{abstract}

Key words : Heat transfer, steady flow, second-order fluid, two enclosed discs, rotating with different angular velocities.

\section{Introduction}

The phenomenon of flow and heat transfer of the fluid between two enclosed rotating discs (enclosed in a cylindrical casing) or shrouded discs has important engineering application as its generalization could be helpful in the study of heat transfer analysis of air cooling of turbine discs ${ }^{[1]}$ and the determination of oil film temperature of pedestal bearing with central feeding of lubricant ${ }^{[2]}$. Soo et $\mathrm{al}^{[3]}$ has investigated the nature of heat transfer from an enclosed rotating disc for viscous flow. Sharma and Agarwal ${ }^{[4]}$ reconsidered this problem using an improved formulation suggested by Sharma ${ }^{[5]}$. Sharma and $\mathrm{Singh}^{[6]}$ have studied the problem of heat transfer in the flow of a second-order fluid between two enclosed rotating discs.

In the present investigations we are concerned with the heat transfer in the steady incompressible flow of a second-order fluid between two enclosed discs rotating with different angular velocities in the following two cases:

(i) When the discs rotate in the same sense with equal angular velocity.

(ii) When both discs rotate with different angular velocities.

\section{Formulation Of The Problem}

The constitutive equation of an incompressible second-order fluid as suggested by Coleman and $\mathrm{Noll}^{[7]}$ can be written as

$$
\tau_{i j}=-p \delta_{i j}+2 \mu_{1} d_{i j}+2 \mu_{2} e_{i j}+4 \mu_{3} c_{i j}
$$

where

$$
\begin{aligned}
d_{i j} & =\frac{1}{2}\left(u_{i, j}+u_{j, i}\right), \\
e_{i j} & =\frac{1}{2}\left(a_{i, j}+a_{j, i}\right)+u_{, i}^{m} u_{m, j} \\
c_{i j} & =d_{i}^{m} d_{m j}
\end{aligned}
$$

where $p$ is the hydrostatic pressure, $\tau_{i j}$ is the stress tensor, $u_{i}$ and $a_{i}$ are velocity and acceleration vector.

The equation (1) together with the momentum equation for no extraneous force

$$
\rho\left(\frac{\partial u_{i}}{\partial t}+u^{m} u_{i, m}\right)=\tau_{i, m}^{m}
$$

and the equation of continuity for incompressible fluid 


$$
u_{, i}^{i}=0
$$

where $\rho$ is the density of the fluid and (,) represents covariant differentiation, forms the set of governing equations. The material constants $\mu_{1}, \mu_{2}$ and $\mu_{3}$ are known as coefficients of Newtonian-viscosity, elasticoviscosity and cross-viscosity respectively, whose values are given by $\mu_{1}=18.5, \mu_{2}=-0.2$ and $\mu_{3}=1.0$ (all expressed in CGS units for $5.46 \%$ solutions of poly-isobutylene in cetane at $30^{\circ} \mathrm{C}$ as suggested by Markowitz ${ }^{[8]}$ )

In a three dimensional cylindrical set of co-ordinate $(r, \theta, z)$, the system consists of two finite discs of radius $r_{s}$ (coinciding with the plane $z=0$ and $z=z_{0}<r_{s}$ ) rotating with different velocities. The lower disc is rotating with angular velocity $\Omega$ whenever the upper disc is rotating with different angular velocity $N \Omega$ in an incompressible second-order fluid forming the part of a co-axial cylindrical casing. The symmetrical radial steady out flow has a small mass rate ' $m$ ' for radial outflow and ' $-m$ ' for radial inflow. The inlet conditions is taken as a simple radial source flow along the $z$-axis starting from radius $r_{0}$. The lower and upper discs are maintained at constant temperature $T_{a}$ and $T_{b}$ respectively.

The energy equation describing the transport of thermal energy is

$$
\rho c_{v} \frac{D T}{D t}=k \nabla^{2} T+\Phi
$$

where $c_{v}, k$ and $\rho$ are the specific heat, thermal conductivity and density of fluid; $T$ is the temperature and $\Phi$ is the viscous dissipations. In equation (5), the first term represents the contribution of convection, the second term is due to viscous dissipation which is given by

$$
\Phi=\tilde{\tau}_{j}^{i} d_{i}^{j}
$$

where $\tilde{\tau}_{j}^{i}$ is the deviatoric stress tensor and the dissipation function $\Phi$ indicates the energy which is dissipated into heat due to friction in the fluid. The boundary conditions on the velocity profile are:

$$
\begin{array}{lllll}
z=0: & u=0, & v=r \Omega, & w=0, & T=T_{a} \\
z=z_{0}: & u=0, & v=N r \Omega, & w=0, & T=T_{b}
\end{array}
$$

where $(u, v, w)$ are the velocity components along the cylindrical system of axis $(r, \theta, z)$. The velocity components for the axisymmetric flow compatible with the continuity criterion can be taken as ${ }^{[6]}$.

$$
\begin{aligned}
& U=-\xi H^{\prime}(\zeta)+\xi^{-1}\left(\frac{R_{m}}{R_{z}}\right) M^{\prime}(\zeta) \\
& V=\xi G(\zeta)+\xi^{-1}\left(\frac{R_{l}}{R_{z}}\right) L(\zeta) \\
& W=2 H(\zeta)
\end{aligned}
$$

where $U=\frac{u}{\Omega z_{0}}, V=\frac{v}{\Omega z_{0}}, W=\frac{w}{\Omega z_{0}}, \quad T^{*}=\left(\frac{c_{v}}{v_{1} \Omega}\right) T, \Phi^{*}=\frac{\Phi}{\mu_{1} \Omega^{2}}, \xi=\frac{r}{z_{0}}$, $\zeta=\frac{z}{z_{0}}$ are dimensionless quantities and $H(\zeta), G(\zeta), L(\zeta), M^{\prime}(\zeta)$ are dimensionless functions of the dimensionless variable $\zeta \cdot R_{m}\left(=\frac{m}{\left(2 \pi \rho z_{0} v_{1}\right)}\right), R_{l}\left(=\frac{l}{\left(2 \pi \rho z_{0} v_{1}\right)}\right)$ are dimensionless numbers to be 
Heat Transfer In The Steady Flow Of A Second-Order Fluid Between Two Enclosed Discs Rotating called Reynolds number of net radial outflow and circulatory flow respectively ( $R_{m}$ is negative for a net radial inflow), $R_{z}\left(=\frac{\Omega z_{0}^{2}}{v_{1}}\right)$ be the flow Reynold's number. The small mass rate ' $m$ ' of the radial outflow is represented by

$$
m=2 \pi \rho \int_{0}^{z_{0}} r u d z
$$

Using expression (8), the boundary conditions transform for $G, L$ and $H$ into the following form:

$$
\begin{aligned}
& \zeta=0 \quad: \quad G=1, \quad L=0, \quad H=0, \quad H^{\prime}=0 \\
& \zeta=1 \quad: \quad G=N, \quad L=0, \quad H=0, \quad H^{\prime}=0
\end{aligned}
$$

The condition on $M$ on the boundaries are obtainable from the equation (9) for $m$ as follows

$$
M(1)-M(0)=1,
$$

which on choosing the discs as streamlines reduces to:

$$
M(1)=1 \quad M(0)=0
$$

Using equation (1) and expression (8) in equation (3) and neglecting the squares and higher powers of $\frac{R_{m}}{R_{z}}$ (assumed small) the dimensionless equation of motion for transverse component of the velocity is-

$$
\begin{aligned}
0=- & 2 \Omega^{2} z_{0} \xi\left(H G^{\prime}-H^{\prime} G\right)-\Omega^{2} z_{0}\left(\frac{R_{m}}{R_{z}}\right)\left(\frac{2 M^{\prime} G}{\xi}\right)-\Omega^{2} z_{0}\left(\frac{R_{l}}{R_{z}}\right)\left(\frac{2 H L^{\prime}}{\xi}\right) \\
& +v_{1} \frac{\Omega}{z_{0}}\left\{\xi G^{\prime \prime}+\left(\frac{R_{l}}{R_{z}}\right)\left(\frac{L^{\prime \prime}}{\xi}\right)\right\}+\left(\frac{2 v_{2}}{z_{0}}\right)\left[( \frac { R _ { l } } { R _ { z } } ) ( \frac { \Omega ^ { 2 } } { \xi } ) \left(H^{\prime \prime} L^{\prime}+H^{\prime \prime \prime} L\right.\right. \\
& \left.+H L^{\prime \prime \prime}+H^{\prime} L^{\prime \prime}\right)+\left(\Omega^{2} \xi\right)\left(H G^{\prime \prime \prime}-H^{\prime \prime} G^{\prime}\right)+\left(\frac{R_{m}}{R_{z}}\right)\left(\frac{2 \Omega^{2}}{\xi}\right)\left(M^{\prime} G^{\prime \prime}\right. \\
& \left.\left.+M^{\prime \prime} G^{\prime}\right)\right]+\left(\frac{2 v_{3} \Omega^{2}}{z_{0}}\right)\left\{\xi\left(H^{\prime} G^{\prime \prime}-H^{\prime \prime} G^{\prime}\right)+\left(\frac{R_{l}}{R_{z}}\right)\left(\frac{1}{\xi}\right)\left(H^{\prime \prime} L^{\prime}+H^{\prime \prime \prime} L\right.\right. \\
& \left.\left.+H^{\prime} L^{\prime \prime}\right)+\left(\frac{R_{m}}{R_{z}}\right)\left(\frac{1}{\xi}\right)\left(2 M^{\prime \prime} G^{\prime}+M^{\prime} G^{\prime \prime}\right)\right\}
\end{aligned}
$$

After eliminating the pressure $p$ from the equation of motion of radial and axial component we get-

$$
\begin{aligned}
&-2 \Omega^{2} z_{0} \xi\left(H H^{\prime \prime \prime}+G G^{\prime}\right)+\left(\frac{R_{m}}{R_{z}}\right)\left(\frac{2 \Omega^{2} z_{0}}{\xi}\right)\left(H^{\prime} M^{\prime \prime}+H M^{\prime \prime \prime}\right)-\left(\frac{R_{l}}{R_{z}}\right) \times \\
& \quad\left(\frac{2 \Omega^{2} z_{0}}{\xi}\right)\left(L G^{\prime}+L^{\prime} G\right)-\left(\frac{v_{1} \Omega}{z_{0}}\right)\left\{\left(\frac{R_{m}}{R_{z}}\right)\left(\frac{M^{i v}}{\xi}\right)-\xi H^{i v}\right\}-\left(\frac{2 v_{2}}{z_{0}}\right) \times \\
& {\left[-\Omega^{2} \xi\left(2 H^{\prime \prime} H^{\prime \prime \prime}+H^{\prime} H^{i v}+H H^{v}+4 G^{\prime} G^{\prime \prime}\right)+\left(\frac{R_{m}}{R_{z}}\right)\left(\frac{\Omega^{2}}{\xi}\right)\left(2 H^{\prime \prime \prime} M^{\prime \prime}\right.\right.} \\
&+\left.H^{i v} M^{\prime}+2 H^{\prime \prime} M^{\prime \prime \prime}+2 H^{\prime} M^{i v}+H M^{v}\right)-\left(\frac{R_{l}}{R_{z}}\right)\left(\frac{2 \Omega^{2}}{\xi}\right)\left(2 L^{\prime} G^{\prime \prime}\right.
\end{aligned}
$$




$$
\begin{aligned}
& \left.\left.+L^{\prime \prime} G^{\prime}+L G^{\prime \prime \prime}\right)\right]-\left(\frac{2 v_{3} \Omega^{2}}{z_{0}}\right)\left\{( \frac { R _ { m } } { R _ { z } } ) ( \frac { 1 } { \xi } ) \left(H^{i v} M^{\prime}+2 H^{\prime \prime \prime} M^{\prime \prime}\right.\right. \\
& \left.+2 H^{\prime \prime} M^{\prime \prime \prime}+H^{\prime} M^{i v}\right)-\left(\frac{R_{l}}{R_{z}}\right)\left(\frac{1}{\xi}\right)\left(3 L^{\prime} G^{\prime \prime}+2 L^{\prime \prime} G^{\prime}+L G^{\prime \prime \prime}\right) \\
& \left.-\xi\left(H^{\prime} H^{i v}+3 G^{\prime} G^{\prime \prime}+2 H^{\prime \prime} H^{\prime \prime \prime}\right)\right\}=0
\end{aligned}
$$

The energy equation (5) take the dimensionless form as:

$U \frac{\partial T^{*}}{\partial \xi}+W \frac{\partial T^{*}}{\partial \zeta}=\frac{1}{P R_{z}}\left(\frac{\partial T^{*}}{\partial \xi^{2}}+\frac{1}{\xi} \frac{\partial T^{*}}{\partial \xi}+\frac{\partial^{2} T^{*}}{\partial \zeta^{2}}\right)+\Phi^{*}$

where $P\left(=\frac{\mu_{1} c_{v}}{k}\right)$ is the prandtl number.

\section{Solution Of The Problem}

On equating the coefficients of $\xi$ and $\frac{1}{\xi}$ from the equations (13) and (14) and assuming $R_{m} \sim R_{z}$ and expanding the function $H, G, L, M$ in the ascending powers of $R_{z}$ (assumed small such that $R_{z}^{3}$ and higher power of $R_{z}$ are neglected) as:

$$
\begin{aligned}
& G(\zeta)=G_{0}(\zeta)+R_{z} G_{1}(\zeta)+R_{z}^{2} G_{2}(\zeta) \\
& L(\zeta)=L_{0}(\zeta)+R_{z} L_{1}(\zeta)+R_{z}^{2} L_{2}(\zeta) \\
& H(\zeta)=H_{0}(\zeta)+R_{z} H_{1}(\zeta)+R_{z}^{2} H_{2}(\zeta) \\
& M(\zeta)=M_{0}(\zeta)+R_{z} M_{1}(\zeta)+R_{z}^{2} M_{2}(\zeta)
\end{aligned}
$$

We get the set of differential equations in terms of $G_{0}, G_{1}, G_{2} ; L_{0}, L_{1}, L_{2} ; H_{0}$, $H_{1}, H_{2} ; M_{0}, M_{1}, M_{2}$ and their derivatives which when integrated subject to the boundary conditions:

$$
\begin{aligned}
& G_{0}(0)=1, G_{1}(0)=0, G_{2}(0)=0 \quad G_{0}(1)=N, G_{1}(1)=0, \quad G_{2}(1)=0 \\
& H_{0}(0)=0, \quad H_{1}(0)=0, \quad H_{2}(0)=0 \quad H_{0}(1)=0, \quad H_{1}(1)=0, \quad H_{2}(1)=0 \\
& H_{0}^{\prime}(0)=0, \quad H_{1}^{\prime}(0)=0, \quad H_{2}^{\prime}(0)=0 \quad H_{0}^{\prime}(1)=0, \quad H_{1}^{\prime}(1)=0, \quad H_{2}^{\prime}(1)=0 \\
& L_{0}(0)=0, \quad L_{1}(0)=0, \quad L_{2}(0)=0 \quad L_{0}(1)=0, \quad L_{1}(1)=0, \quad L_{2}(1)=0 \\
& M_{0}(0)=0, \quad M_{1}(0)=0, \quad M_{2}(0)=0 \quad M_{0}(1)=0, \quad M_{1}(1)=0, \quad M_{2}(1)=0 \\
& M_{0}^{\prime}(0)=0, M_{1}^{\prime}(0)=0, M_{2}^{\prime}(0)=0 \quad M_{0}^{\prime}(1)=0, M_{1}^{\prime}(1)=0, M_{2}^{\prime}(1)=0
\end{aligned}
$$

Gives the values of $G_{0}, G_{1}, G_{2} ; H_{0}, H_{1}, H_{2} ; L_{0}, L_{1}, L_{2} ; M_{0}, M_{1}, M_{2}$.

Hence we get the values of the functions $H, G, L, M$ calculated under the assumption of smallness of $\left(\frac{R_{m}}{R_{z}}\right)$ and equivalence of the order of $R_{m}$ and $R_{l}$ correct to the square of flow Reynolds number $R_{z}$, and first power of $\left(\frac{R_{m}}{R_{z}}\right)$ are: 


$$
\begin{aligned}
& H=\left(\frac{R_{z}}{60}\right)\left[\zeta^{5}-5 \zeta^{4}+7 \zeta^{3}-3 \zeta^{2}+N^{2}\left(\zeta^{5}-3 \zeta^{3}+2 \zeta^{2}\right)\right. \\
& \left.-N\left(2 \zeta^{5}-5 \zeta^{4}+4 \zeta^{3}-\zeta^{2}\right)\right] \\
& G=1+(N-1) \zeta+\left(\frac{R_{z}^{2}}{6300}\right)\left[20 \zeta^{7}-140 \zeta^{6}+357 \zeta^{5}-420 \zeta^{4}+210 \zeta^{3}-27 \zeta\right. \\
& -N^{3}\left(20 \zeta^{7}-63 \zeta^{5}+35 \zeta^{4}+8 \zeta\right)-N\left(60 \zeta^{7}-280 \zeta^{6}+441 \zeta^{5}-280 \zeta^{4}\right. \\
& \left.\left.+70 \zeta^{3}-11 \zeta\right)+N^{2}\left(60 \zeta^{7}-140 \zeta^{6}+21 \zeta^{5}+175 \zeta^{4}-140 \zeta^{3}+24 \zeta\right)\right] \\
& +\left(\frac{R_{z}^{2}}{30}\right)\left(\tau_{1}+\tau_{2}\right)\left[N^{3}\left(\zeta^{5}-3 \zeta^{3}+2 \zeta^{2}\right)-\left(\zeta^{5}-5 \zeta^{4}+7 \zeta^{3}-3 \zeta^{2}\right)\right. \\
& \left.+N\left(3 \zeta^{5}-10 \zeta^{4}+11 \zeta^{3}-4 \zeta^{2}\right)-N^{2}\left(3 \zeta^{5}-5 \zeta^{4}+\zeta^{3}+\zeta^{2}\right)\right] \\
& L=\left(\frac{R_{m}}{R_{l}}\right) R_{z}\left[\frac { 1 } { 5 } \left\{\left(3 \zeta^{5}-10 \zeta^{4}+10 \zeta^{3}-3 \zeta\right)-N\left(3 \zeta^{5}-5 \zeta^{4}+2 \zeta\right)\right.\right. \\
& \left.\left.+20\left(\tau_{1}+\tau_{2}\right)(N-1)\left(2 \zeta^{3}-3 \zeta^{2}+\zeta\right)\right\}\right] \\
& M^{\prime}=6\left(\zeta-\zeta^{2}\right)+\left(\frac{R_{z}^{2}}{4200}\right)\left[60 \zeta^{8}-300 \zeta^{7}+588 \zeta^{6}-294 \zeta^{5}-630 \zeta^{4}+840 \zeta^{3}\right.
\end{aligned}
$$$$
-263 \zeta^{2}-\zeta+N^{2}\left(60 \zeta^{8}-180 \zeta^{7}+168 \zeta^{6}-294 \zeta^{5}+420 \zeta^{4}-263 \zeta^{2}\right.
$$$$
+89 \zeta)-2 N\left(60 \zeta^{8}-240 \zeta^{7}+238 \zeta^{6}+126 \zeta^{5}-105 \zeta^{4}-280 \zeta^{3}+247 \zeta^{2}\right.
$$$$
-46 \zeta)]-\left(\frac{R_{z}^{2}}{525}\right)\left(\tau_{1}+\tau_{2}\right)\left[105 \zeta^{6}-595 \zeta^{4}+1190 \zeta^{3}-939 \zeta^{2}+239 \zeta\right.
$$$$
+N^{2}\left(105 \zeta^{6}-630 \zeta^{5}+980 \zeta^{4}-910 \zeta^{3}+636 \zeta^{2}-181 \zeta\right)-N\left(210 \zeta^{6}\right.
$$$$
\left.\left.-630 \zeta^{5}+385 \zeta^{4}+280 \zeta^{3}-303 \zeta^{2}+58 \zeta\right)\right]
$$$$
+\frac{8}{5} R_{z}^{2}\left(\tau_{1}+\tau_{2}\right)^{2}(N-1)^{2}\left(5 \zeta^{4}-10 \zeta^{3}+6 \zeta^{2}-\zeta\right)
$$

where $v_{1}=\frac{\mu_{1}}{\rho}, v_{2}=\frac{\mu_{2}}{\rho}$ and $v_{3}=\frac{\mu_{3}}{\rho}$ are the kinematic-viscosity, elastico- viscosity and crossviscosity respectively, $\tau_{1}\left(=\frac{v_{2}}{z_{0}^{2}}\right)$ and $\tau_{2}\left(=\frac{v_{3}}{z_{0}^{2}}\right)$ are the elastico-viscous and cross-viscous dimensionless parameters. 
Using (8) in equation (15) and neglecting terms of order $\left(\frac{R_{m}}{R_{z}}\right)^{2}$ and higher, we get

$$
\begin{aligned}
& {\left[\left(-\xi H^{\prime}+\frac{1}{\xi}\left(\frac{R_{m}}{R_{z}}\right) M^{\prime}\right) \frac{\partial T^{*}}{\partial \xi}+2 H \frac{\partial T^{*}}{\partial \zeta}\right]} \\
& =\frac{1}{P R_{z}}\left[\frac{\partial^{2} T^{*}}{\partial \xi^{2}}+\frac{1}{\xi} \frac{\partial T^{*}}{\partial \xi}+\frac{\partial^{2} T^{*}}{\partial \zeta^{2}}\right]+\frac{1}{2}\left[24 H^{\prime 2}+4 \frac{R_{m}}{R_{z}}\left(\frac{R_{l}}{R_{m}} G^{\prime} L^{\prime}-H^{\prime \prime} M^{\prime \prime}\right)\right. \\
& +48 \tau_{1} R_{z}\left(H^{\prime 3}+H H^{\prime} H^{\prime \prime}\right)+2 R_{m} \tau_{1}\left\{\frac { R _ { l } } { R _ { m } } \left(6 H^{\prime} L^{\prime} G^{\prime}+6 L G^{\prime} H^{\prime \prime}+2 H G^{\prime} L^{\prime \prime}\right.\right. \\
& \left.\left.+2 H L^{\prime} G^{\prime \prime}\right)+\left(-6 H^{\prime} H^{\prime \prime} M^{\prime \prime}-2 H H^{\prime \prime} M^{\prime \prime}-2 M^{\prime} H^{\prime \prime 2}-2 H M^{\prime \prime} H^{\prime \prime \prime}+4 M^{\prime} G^{\prime 2}\right)\right\} \\
& +48 \tau_{2} R_{z^{\prime}} H^{\prime 3}+2 R_{m^{2}} \tau_{2}\left\{\left(-3 M^{\prime} H^{\prime \prime 2}-6 H^{\prime} H^{\prime \prime} M^{\prime \prime}+3 M^{\prime} G^{\prime 2}\right)+\frac{R_{l}}{R_{m}}\left(6 H^{\prime} L^{\prime} G^{\prime}\right.\right. \\
& \left.\left.\left.+6 L G^{\prime} H^{\prime \prime}\right)\right\}\right]+\frac{1}{2} \xi^{2}\left[2\left(H^{\prime \prime 2}+G^{\prime 2}\right)+\tau_{1} R_{z}\left(4 H^{\prime} H^{\prime \prime 2}+4 H^{\prime} G^{\prime 2}\right.\right. \\
& \left.\left.+4 H H^{\prime \prime} H^{\prime \prime \prime}+4 H G^{\prime} G^{\prime \prime}\right)+6 \tau_{2} R_{z}\left(H^{\prime} H^{\prime \prime 2}+H^{\prime} G^{\prime 2}\right)\right] \quad \ldots(19)
\end{aligned}
$$

The appropriate form for $T^{*}$ as suggested from equation (19) is

$$
T^{*}=T_{a}^{*}+\phi(\zeta)+\xi^{2} \psi(\zeta)
$$

where $T_{a}^{*}$ denotes the dimensionless temperature on the lower disc and $\phi, \psi$ are the dimensionless functions of $\zeta$. Substituting equation (20) into (19) and equating the coefficients of $\xi^{2}$ and terms independent of $\xi$ on both sides of the equation thus obtained, we get

$$
\begin{aligned}
& \frac{1}{P}\left(4 \psi+\phi^{\prime \prime}\right)=2 R_{m}\left[M^{\prime} \psi-\left\{\left(\frac{R_{l}}{R_{m}}\right) G^{\prime} L^{\prime}-H^{\prime \prime} M^{\prime \prime}\right\}\right]+2 R_{z}\left[H \phi^{\prime}-6 H^{\prime 2}\right. \\
& -\frac{1}{2} \tau_{1} R_{m}\left\{\left(\frac{R_{l}}{R_{m}}\right)\left(6 H^{\prime} L^{\prime} G^{\prime}+6 L G^{\prime} H^{\prime \prime}+2 H G^{\prime} L^{\prime \prime}+2 H L^{\prime} G^{\prime \prime}\right)\right. \\
& \left.+\left(-6 H^{\prime} H^{\prime \prime} M^{\prime \prime}-2 H H^{\prime \prime} M^{\prime \prime}-2 M^{\prime} H^{\prime \prime 2}-2 H M^{\prime \prime} H^{\prime \prime \prime}+4 M^{\prime} G^{\prime 2}\right)\right\} \\
& -\frac{1}{2} \tau_{2} R_{m}\left\{\left(-3 M^{\prime} H^{\prime \prime 2}-6 H^{\prime} H^{\prime \prime} M^{\prime \prime}+3 M^{\prime} G^{\prime 2}\right)+\left(\frac{R_{l}}{R_{m}}\right)\left(6 H^{\prime} L^{\prime} G^{\prime}\right.\right.
\end{aligned}
$$




$$
\begin{gathered}
\left.\left.\left.+6 L G^{\prime} H^{\prime \prime}\right)\right\}\right]-24 R_{z}^{2}\left[\tau_{1}\left(H^{\prime 3}+H H^{\prime} H^{\prime \prime}\right)+\tau_{2} H^{\prime 3}\right] \\
\frac{1}{P} \psi^{\prime \prime}=R_{z}\left[-2 H^{\prime} \psi+2 H \psi^{\prime}-H^{\prime \prime 2}-{G^{\prime}}^{2}\right]-R_{z}^{2}\left[2 \tau _ { 1 } \left(H^{\prime} H^{\prime \prime 2}+H^{\prime} G^{\prime 2}\right.\right. \\
\left.\left.+H H^{\prime \prime} H^{\prime \prime \prime}+H G^{\prime} G^{\prime \prime}\right)+3 \tau_{2}\left(H^{\prime} H^{\prime \prime}{ }^{2}+H^{\prime} G^{\prime 2}\right)\right] . .(22)
\end{gathered}
$$

The expressions for $H, G, L$ and $M^{\prime}$ have been considered upto $\left(R_{z}\right)^{2}$ and hence the appropriate forms for $\phi$ and $\psi$ should be as follows:

$$
\left.\begin{array}{c}
\phi=\phi_{0}+R_{z} \phi_{1}+R_{z}^{2} \phi_{2} \\
\psi=\psi_{0}+R_{z} \psi_{1}+R_{z}^{2} \psi_{2}
\end{array}\right\}
$$

where $\phi_{0}, \psi_{0}$ etc. are functions of $\zeta$.

The boundary conditions on the temperature in terms of $\phi_{0}, \psi_{0}$ etc. can be written as:

$$
\begin{aligned}
& \zeta=0: \phi_{0}=0, \quad \phi_{1}=0, \quad \phi_{2}=0 ; \quad \psi_{0}=0, \quad \psi_{1}=0, \quad \psi_{2}=0 \\
& \zeta=1: \phi_{0}=S, \quad \phi_{1}=0, \quad \phi_{2}=0 ; \quad \psi_{0}=0, \quad \psi_{1}=0, \quad \psi_{2}=0
\end{aligned}
$$

where $S=\frac{\left(T_{b}-T_{a}\right) c_{v}}{\left(v_{1} \Omega\right)}$

Substituting (23) into (21) and (22) and equating the terms independent of $R_{z}$, coefficient of $R_{z}$ and $R_{z}^{2}$ on both sides of the resulting equations, we get a set of equations containing $\phi_{0}, \psi_{0}$ etc. and their derivatives. The solution of this set of equations satisfying the boundary condition (24) is given by:

$$
\begin{aligned}
& \psi_{0}=0, \quad \psi_{1}=\frac{1}{2} P(N-1)^{2} \zeta(1-\zeta), \quad \psi_{2}=0 \\
& \phi_{0}=S \zeta \\
& \phi_{1}=\frac{1}{6} P(N-1)^{2}\left(\zeta^{4}-2 \zeta^{3}+\zeta\right)+P R_{m}\left[\frac{1}{10} P(N-1)^{2}\left(2 \zeta^{6}-6 \zeta^{5}+5 \zeta^{4}-\zeta\right)\right. \\
& -\frac{1}{15}\left\{\zeta^{6}-9 \zeta^{5}+21 \zeta^{4}-27 \zeta^{3}+18 \zeta^{2}-4 \zeta+N^{2}\left(\zeta^{6}+3 \zeta^{5}-9 \zeta^{4}+13 \zeta^{3}\right.\right. \\
& \left.\left.-12 \zeta^{2}+4 \zeta\right)-2 N\left(\zeta^{6}-3 \zeta^{5}+6 \zeta^{4}-7 \zeta^{3}+3 \zeta^{2}\right)\right\}-\left\{2 \tau _ { 1 } \left(\zeta^{4}-2 \zeta^{3}\right.\right. \\
& \left.\left.\left.+2 \zeta^{2}-\zeta\right)+\frac{1}{2} \tau_{2}\left(5 \zeta^{4}-10 \zeta^{3}+8 \zeta^{2}-3 \zeta\right)\right\}(N-1)^{2}\right] \\
& \phi_{2}=\frac{P S}{(12600)}\left[\left(10 \zeta^{7}-70 \zeta^{6}+147 \zeta^{5}-105 \zeta^{4}+18 \zeta\right)+N^{2}\left(10 \zeta^{7}-63 \zeta^{5}\right.\right. \\
& \left.\left.+70 \zeta^{4}-17 \zeta\right)-N\left(20 \zeta^{7}-70 \zeta^{6}+84 \zeta^{5}-35 \zeta^{4}+\zeta\right)\right]
\end{aligned}
$$

Using equations (20), (23) and (25) we get the solution of equation (19) the dimensionless form of the temperature as: 


$$
\begin{aligned}
\frac{T-T_{a}}{T_{b}-T_{a}} & =\frac{T^{*}-T_{a}^{*}}{T_{b}^{*}-T_{a}^{*}}=\zeta+\left(\frac{P R_{z}^{2}}{12600}\right)\left\{10 \zeta^{7}-70 \zeta^{6}+147 \zeta^{5}-105 \zeta^{4}+18 \zeta\right. \\
& +N^{2}\left(10 \zeta^{7}-63 \zeta^{5}+70 \zeta^{4}-17 \zeta\right)-N\left(20 \zeta^{7}-70 \zeta^{6}+84 \zeta^{5}-35 \zeta^{4}\right. \\
& +\zeta)\}+E P\left[\frac{1}{6}(N-1)^{2}\left(\zeta^{4}-2 \zeta^{3}+\zeta\right)+\frac{R_{m}}{30}\left\{3 P ( N - 1 ) ^ { 2 } \left(2 \zeta^{6}\right.\right.\right. \\
& \left.-6 \zeta^{5}+5 \zeta^{4}-\zeta\right)-2\left\{\zeta^{6}-9 \zeta^{5}+21 \zeta^{4}-27 \zeta^{3}+18 \zeta^{2}-4 \zeta^{2}-13 \zeta^{3}-12 \zeta^{2}+4 \zeta\right)+2 N\left(-\zeta^{6}+3 \zeta^{5}-6 \zeta^{4}\right. \\
& +N^{2}\left(\zeta^{6}+3 \zeta^{5}-9 \zeta^{4}+10 \zeta^{3}\right. \\
& \left.\left.+7 \zeta^{3}-3 \zeta^{2}\right)\right\}-\left\{60 \tau_{1}\left(\zeta^{4}-2 \zeta^{3}+2 \zeta^{2}-\zeta\right)+15 \tau_{2}\left(5 \zeta^{4}-10 \zeta^{3}\right.\right. \\
& \left.\left.\left.\left.+8 \zeta^{2}-3 \zeta\right)\right\}(N-1)^{2}\right\}+\frac{1}{2} \xi^{2}(N-1)^{2} \zeta(1-\zeta)\right] \quad \ldots(26)
\end{aligned}
$$

where $E\left(=\frac{\Omega z_{0}^{2}}{c_{v}\left(T_{b}-T_{a}\right)}\right)$ is the Eckert number.

The amount of heat transfer from the lower and upper discs are

$$
Q_{a}=\frac{1}{\pi\left(\xi^{2}-\xi_{0}^{2}\right)} \int_{\xi_{0}}^{\xi} 2 \pi \xi q_{a} d \xi
$$

and

$$
Q_{b}=\frac{1}{\pi\left(\xi^{2}-\xi_{0}^{2}\right)} \int_{\xi_{0}}^{\xi} 2 \pi \xi q_{b} d \xi
$$

respectively.

$q_{a}\left(=-\left.\left(\frac{k}{z_{0}}\right) \frac{\partial T}{\partial \zeta}\right|_{\zeta=0}\right)$ and $q_{b}\left(=-\left.\left(\frac{k}{z_{0}}\right) \frac{\partial T}{\partial \zeta}\right|_{\zeta=1}\right)$ are heat fluxes on the lower and upper discs. Therefore the average Nusselt numbers on the lower and upper discs are

$$
\begin{aligned}
N u_{a}= & \frac{Q_{a} z_{0}}{\left(T_{b}-T_{a}\right) k} \\
= & -\left[1+\frac{P R_{z}^{2}}{12600}\left(18-17 N^{2}-N\right)+E P\left\{\frac{1}{6}(N-1)^{2}\right.\right. \\
& +\frac{R_{m}}{30}\left\{-3 P(N-1)^{2}+8-8 N^{2}+60 \tau_{1}(N-1)^{2}+45 \tau_{2}(N-1)^{2}\right\} \\
& \left.\left.+\frac{1}{4}\left(\xi^{2}+\xi_{0}^{2}\right)(N-1)^{2}\right\}\right] \quad \ldots(29) \\
N u_{b}= & \frac{Q_{b} z_{0}}{\left(T_{b}-T_{a}\right) k}=-\left[1+\frac{P R_{z}^{2}}{12600}\left(-17+18 N^{2}-N\right)+E P\left\{-\frac{1}{6}(N-1)^{2}\right.\right.
\end{aligned}
$$


Heat Transfer In The Steady Flow Of A Second-Order Fluid Between Two Enclosed Discs Rotating

$$
\begin{aligned}
& +\frac{R_{m}}{30}\left\{3 P(N-1)^{2}+8-8 N^{2}-60 \tau(N-1)^{2}-45 \tau_{2}(N-1)^{2}\right\} \\
& \left.\left.-\frac{1}{4}\left(\xi^{2}+\xi_{0}^{2}\right)(N-1)^{2}\right\}\right]
\end{aligned}
$$

\section{Results And Discussion}

The dimensionless form of the radii at which there is no recirculation for the cases of net radial outflow $\left(R_{m}>0\right)$ and net radial inflow $\left(R_{m}<0\right)$ respectively, satisfying the following conditions

$$
\begin{aligned}
& \text { I } \quad R_{m}>0 \quad\left(\frac{\partial U}{\partial \zeta}\right)_{\zeta=0} \geq 0, \quad\left(\frac{\partial U}{\partial \zeta}\right)_{\zeta=1} \leq 0 \\
& \text { II } \quad R_{m}\left(=-R_{n}<0\right) \quad\left(\frac{\partial U}{\partial \zeta}\right)_{\zeta=0} \leq 0, \quad\left(\frac{\partial U}{\partial \zeta}\right)_{\zeta=1} \geq 0
\end{aligned}
$$

Case I: When both the discs rotate in the same sense with equal angular velocity $(N=1)$ :

It is evident from equation (26) that there is no effect of the second-order parameter $\tau_{1}, \tau_{2}$ and $\xi, E, R_{m}$ on the temperature profile. It is also observed that the temperature variation with $\zeta$ is linear. The average Nusselt numbers in this case are constants for the constant value of $R_{z}, P$.

Case II: When both the discs rotate with different angular velocities:

The maximum value of the dimensionless radii $\xi$ for no-recirculation viz. $\xi_{1}$ (for $m>0$ ) and $\xi_{2}$ (for $m<0$ ) are obtained from the condition (31) and (32) respectively as:

$$
\begin{aligned}
& \frac{\xi_{1}^{2}}{R_{m}}=\frac{25200-R_{z}^{2}\left[6720(N-1)^{2} \tau^{2}+8 \tau\left(239-181 N^{2}-58 N\right)+1-89 N^{2}-92 N\right]}{70 R_{z}^{2}\left(4 N^{2}+2 N-6\right)} \\
& \frac{\xi_{2}^{2}}{R_{n}}=\frac{25200-R_{z}^{2}\left[6720(N-1)^{2} \tau^{2}-8 \tau\left(181-239 N^{2}+58 N\right)+N^{2}-92 N-89\right]}{70 R_{z}^{2}\left(-4+6 N^{2}-2 N\right)}
\end{aligned}
$$

where $\tau=\tau_{1}+\tau_{2}$ and $R_{m}=-R_{n}\left(R_{n}>0\right)$ for $m<0$.

The behaviour of the dimensionless temperature $\frac{\left(T-T_{a}\right)}{\left(T_{b}-T_{a}\right)}$ with $\zeta$ at $P=20$, $E=0.02, R_{z}=0.5, N=2$ for different values of $\tau_{2}=0,1,2$ in cases of $R_{m}(=-0.02)<0 ; R_{m}(=0.02)>0$ when $\xi=20$ and $\xi=1$ is represented through figure- 1 and figure-2 respectively.

Table-1 Variation of Temperature with $\zeta$ for different values of $\tau_{2}$ at $N=2$ in Recirculation region $(\xi=20)$ in case of $R_{m}<0$ and $R_{m}>0$

\begin{tabular}{|r|r|r|r|r|r|r|}
\hline & \multicolumn{3}{|c|}{$R_{m}=-0.02$} & \multicolumn{3}{c|}{$R_{m}=0.02$} \\
\hline$\zeta$ & \multicolumn{1}{|c|}{$\tau_{2}=0$} & \multicolumn{1}{|c|}{$\tau_{2}=1$} & $\tau_{2}=2$ & $\tau_{2}=0$ & $\tau_{2}=1$ & $\tau_{2}=2$ \\
\hline 0 & 0 & 0 & 0 & 0 & 0 & 0 \\
\hline 0.1 & 7.30652 & 7.305394 & 7.304267 & 7.30245 & 7.303577 & 7.304704 \\
\hline 0.2 & 13.012049 & 13.01018 & 13.008311 & 13.004689 & 13.006557 & 13.008427 \\
\hline 0.3 & 17.116175 & 17.113848 & 17.111523 & 17.106415 & 17.108742 & 17.111069 \\
\hline 0.4 & 19.618626 & 19.616053 & 19.613482 & 19.607544 & 19.610117 & 19.61269 \\
\hline 0.5 & 20.519262 & 20.516613 & 20.513964 & 20.50808 & 20.510729 & 20.51338 \\
\hline 0.6 & 19.818079 & 19.815506 & 19.812933 & 19.808004 & 19.810577 & 19.81315 \\
\hline
\end{tabular}


Heat Transfer In The Steady Flow Of A Second-Order Fluid Between Two Enclosed Discs Rotating

\begin{tabular}{|r|r|r|r|r|r|r|}
0.7 & 17.515198 & 17.512873 & 17.510546 & 17.50725 & 17.509577 & 17.511904 \\
\hline 0.8 & 13.610898 & 13.609029 & 13.607161 & 13.6057 & 13.60757 & 13.609438 \\
\hline 0.9 & 8.105617 & 8.104489 & 8.103363 & 8.103265 & 8.104391 & 8.105518 \\
\hline 1 & 1 & 1 & 1 & 1 & 1 & 1 \\
\hline
\end{tabular}

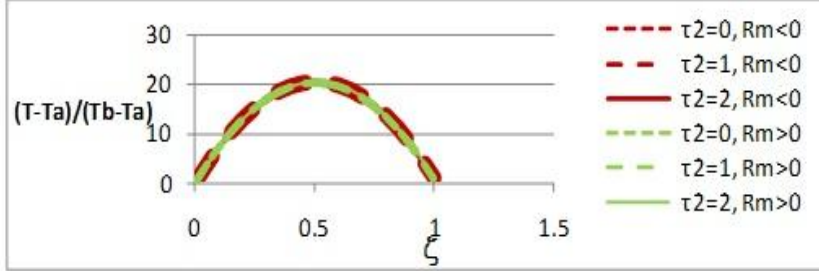

Figure-1 Variation of Temperature with $\zeta$ for different values of $\tau_{2}$ at $N=2$ in Recirculation region $(\xi=20)$ in case of $R_{m}<0$ and $R_{m}>0$

It is clear from the numerical values of the temperature that the temperature decreases with an increase in $\tau_{2}$ in case of net radial inflow $\left(R_{m}<0\right)$ whenever increases in case of net radial outflow $\left(R_{m}>0\right)$ through out the gap length in both the figures-1 $(\xi=20)$ and figure-2 $(\xi=1)$. Due to small increments in temperature with respect to $\tau_{2}$ the branches of the graphs are overlapping in both the figures. In figure-1 the temperature is maximum at the middle of the gap length and minimum (equal to zero) at the lower disc. Temperature is increasing near the lower disc and after attaining its maximum value in the middle it starts decreasing and attains the value 1 on the upper disc whenever in figure- 2 it is zero at the lower disc and attains its maximum value 1 on the upper disc.

Table-2 Variation of Temperature with $\zeta$ for different values of $\tau_{2}$ at $N=2$

in No-Recirculation region $(\xi=1)$ in case of $R_{m}<0$ and $R_{m}>0$

\begin{tabular}{|r|r|r|r|r|r|r|}
\hline & \multicolumn{3}{|c|}{$R_{m}=-0.02$} & \multicolumn{3}{c|}{$R_{m}=0.02$} \\
\hline \multicolumn{1}{r|r|r|r|r|r|r|}{$\tau_{2}=0$} & \multicolumn{1}{|c|}{$\tau_{2}=1$} & \multicolumn{1}{|c|}{$\tau_{2}=2$} & $\tau_{2}=0$ & $\tau_{2}=1$ & \multicolumn{1}{c|}{$\tau_{2}=2$} \\
\hline 0 & 0 & 0 & 0 & 0 & 0 & 0 \\
\hline 0.1 & 0.12452 & 0.123864 & 0.123209 & 0.12045 & 0.121106 & 0.121762 \\
\hline 0.2 & 0.244049 & 0.243071 & 0.242093 & 0.236689 & 0.237667 & 0.238645 \\
\hline 0.3 & 0.358175 & 0.357068 & 0.355961 & 0.348414 & 0.349522 & 0.350629 \\
\hline 0.4 & 0.466627 & 0.465482 & 0.464338 & 0.455545 & 0.45669 & 0.457834 \\
\hline 0.5 & 0.569264 & 0.568114 & 0.566964 & 0.55808 & 0.55923 & 0.56038 \\
\hline 0.6 & 0.666079 & 0.664935 & 0.66379 & 0.656005 & 0.65715 & 0.658294 \\
\hline 0.7 & 0.757201 & 0.756093 & 0.754986 & 0.749251 & 0.750358 & 0.751465 \\
\hline 0.8 & 0.842902 & 0.841924 & 0.840946 & 0.837704 & 0.838682 & 0.83966 \\
\hline 0.9 & 0.923622 & 0.922967 & 0.922311 & 0.921271 & 0.921927 & 0.922583 \\
\hline 1 & 1 & 1 & 1 & 1 & & 1 \\
\hline
\end{tabular}

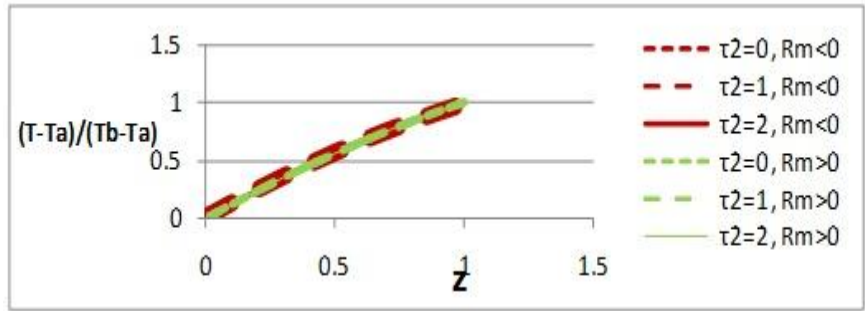

Figure-2 Variation of Temperature with $\zeta$ for different values of $\tau_{2}$ at $N=2$

in No- Recirculation region $(\xi=1)$ in case of $R_{m}<0$ and $R_{m}>0$

In figure-3 $(\xi=20)$ and figure-4 $(\xi=1)$, the value of the temperature at $N=1$ is equal to $\zeta$ for $R_{m}<0$ and $R_{m}>0$. At $P=20, E=0.02, R_{z}=0.5, \tau_{2}=2$ for different values 
Heat Transfer In The Steady Flow Of A Second-Order Fluid Between Two Enclosed Discs Rotating of $N=1,2,3$ the temperature is increasing with an increase in the value of the ratio of angular velocities in case of net radial inflow and outflow in both the figures 3 and 4. The graph of the temperature is linear at $N=1$ with minimum at lower disc and maximum at the upper disc. At $N=2$ and $N=3$, the temperature is maximum in the middle of the gap length in figure- 3 and maximum on the upper disc in figure- 4 .

Table-3 Variation of Temperature with $\zeta$ for different values of $N$ at $\tau_{2}=2$ in

Recirculation region $(\xi=20)$ in case of $R_{m}<0$ and $R_{m}>0$

\begin{tabular}{|c|c|c|c|c|c|c|}
\hline & \multicolumn{3}{|c|}{$R_{m}=-0.02$} & \multicolumn{3}{|c|}{$R_{m}=0.02$} \\
\hline$\zeta$ & $N=1$ & $N=2$ & $N=3$ & $N=1$ & $N=2$ & $N=3$ \\
\hline 0 & 0 & 0 & 0 & 0 & 0 & 0 \\
\hline 0.1 & 0.1 & 7.304267 & 28.923027 & 0.1 & 7.304704 & 28.918385 \\
\hline 0.2 & 0.2 & 13.008311 & 51.445034 & 0.2 & 13.008427 & 51.432682 \\
\hline 0.3 & 0.3 & 17.111523 & 67.562813 & 0.3 & 17.111069 & 67.542694 \\
\hline 0.4 & 0.4 & 19.613482 & 77.274048 & 0.4 & 19.61269 & 77.248703 \\
\hline 0.5 & 0.5 & 20.513964 & 80.5774 & 0.5 & 20.51338 & 80.551071 \\
\hline 0.6 & 0.6 & 19.812933 & 77.472527 & 0.6 & 19.81315 & 77.449875 \\
\hline 0.7 & 0.7 & 17.510546 & 67.960114 & 0.7 & 17.511904 & 67.944824 \\
\hline 0.8 & 0.8 & 13.607161 & 52.041874 & 0.8 & 13.609438 & 52.035286 \\
\hline 0.9 & 0.9 & 8.103363 & 29.720556 & 0.9 & 8.105518 & 29.720497 \\
\hline 1 & 1 & 1 & 1 & 1 & 1 & 1 \\
\hline
\end{tabular}

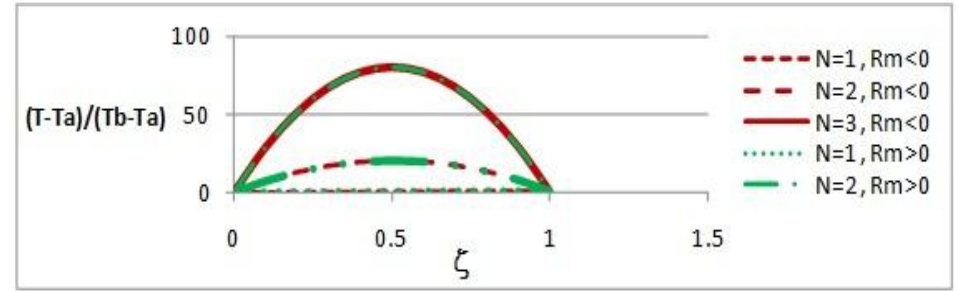

Figure-3 Variation of Temperature with $\zeta$ for different values of $N$ at $\tau_{2}=2$

in Recirculation region $(\xi=20)$ in case of $R_{m}<0$ and $R_{m}>0$

Table-4 Variation of Temperature with $\zeta$ for different values of $N$ at $\tau_{2}=2$

in No- Recirculation region $(\xi=1)$ in case of $R_{m}>0$ and $R_{m}<0$

\begin{tabular}{|r|r|r|r|r|r|r|}
\hline & \multicolumn{3}{|c|}{$R_{m}=-0.02$} & \multicolumn{3}{c|}{$R_{m}=0.02$} \\
\hline$\zeta$ & $N=1$ & $N=2$ & \multicolumn{1}{|c|}{$N=3$} & $N=1$ & $N=2$ & $N=3$ \\
\hline 0 & 0 & 0 & 0 & 0 & 0 & 0 \\
\hline 0.1 & 0.1 & 0.123209 & 0.195027 & 0.1 & 0.121762 & 0.190386 \\
\hline 0.2 & 0.2 & 0.242093 & 0.373036 & 0.2 & 0.238645 & 0.360683 \\
\hline 0.3 & 0.3 & 0.355961 & 0.530814 & 0.3 & 0.350629 & 0.510691 \\
\hline 0.4 & 0.4 & 0.464338 & 0.66605 & 0.4 & 0.457834 & 0.640706 \\
\hline 0.5 & 0.5 & 0.566964 & 0.777403 & 0.5 & 0.56038 & 0.751069 \\
\hline 0.6 & 0.6 & 0.66379 & 0.864532 & 0.6 & 0.658294 & 0.841874 \\
\hline 0.7 & 0.7 & 0.754986 & 0.928122 & 0.7 & 0.751465 & 0.912831 \\
\hline 0.8 & 0.8 & 0.840946 & 0.969887 & 0.8 & 0.83966 & 0.963302 \\
\hline 0.9 & 0.9 & 0.922311 & 0.992581 & 0.9 & 0.922583 & 0.992523 \\
\hline 1 & 1 & 1 & & 1 & & 1 \\
\hline
\end{tabular}




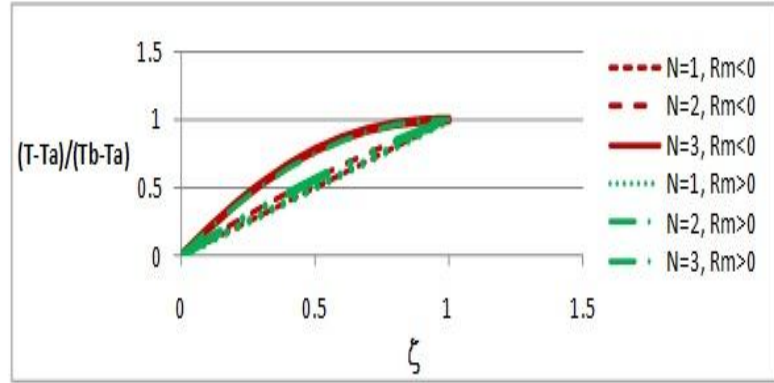

Figure-4 Variation of Temperature with $\zeta$ for different values of $N$ at $\tau_{2}=2$

in No- Recirculation region $(\xi=1)$ in case of $R_{m}<0$ and $R_{m}>0$

Figure-5 represents the variation of $N u_{a}$ (Nusselt number at the lower disc) with $\xi$ at $P=20, E=0.02, R_{z}=0.5, \xi_{0}=5$ for different values of $\tau_{2}=0,1,2$. The average Nusselt number $\left(N u_{a}\right)$ on the lower disc is negative throughout the entire radial region and increases with an increase in $\tau_{2}$ in case of $R_{m}<0$ whenever decreases in case of $R_{m}>0$. It is also clear that $N u_{a}$ is decreasing with an increase in $\xi$ in both the cases $\left(R_{m}>0\right.$ and $\left.R_{m}<0\right)$. The behaviour of $N u_{b}$ (average Nusselt number on the upper disc) in figure-6 is just reverse to that of $N u_{a}$ (see figure-5). $N u_{b}$ is positive throughout the entire radial region and attains its minimum value at the lower disc and maximum at the upper disc.

Table-5 Variation of $N u_{a}$ with $\xi$ for different values of $\tau_{2}$ at $N=2, \xi_{0}=5$ in case of $R_{m}<0$ and $R_{m}>0$

\begin{tabular}{|r|l|l|l|l|l|l|}
\hline & \multicolumn{3}{|c|}{$R_{m}=-0.02$} & \multicolumn{3}{c|}{$R_{m}=0.02$} \\
\hline$\xi$ & $\tau_{2}=0$ & $\tau_{2}=1$ & $\tau_{2}=2$ & $\tau_{2}=0$ & $\tau_{2}=1$ & $\tau_{2}=2$ \\
\hline 0 & -3.56843 & -3.55963 & -3.55083 & -3.52363 & -3.53243 & -3.54123 \\
\hline 1 & -3.66843 & -3.65963 & -3.65083 & -3.62363 & -3.63243 & -3.64123 \\
\hline 2 & -3.96843 & -3.95963 & -3.95083 & -3.92363 & -3.93243 & -3.94123 \\
\hline 3 & -4.46843 & -4.45963 & -4.45083 & -4.42363 & -4.43243 & -4.44123 \\
\hline 4 & -5.16843 & -5.15963 & -5.15083 & -5.12363 & -5.13243 & -5.14123 \\
\hline 5 & -6.06843 & -6.05963 & -6.05083 & -6.02363 & -6.03243 & -6.04123 \\
\hline 6 & -7.16843 & -7.15963 & -7.15083 & -7.12363 & -7.13243 & -7.14123 \\
\hline 7 & -8.46843 & -8.45963 & -8.45083 & -8.42363 & -8.43243 & -8.44123 \\
\hline 8 & -9.96843 & -9.95963 & -9.95083 & -9.92363 & -9.93243 & -9.94123 \\
\hline 9 & -11.6684 & -11.6596 & -11.6508 & -11.6236 & -11.6324 & -11.6412 \\
\hline 10 & -13.5684 & -13.5596 & -13.5508 & -13.5236 & -13.5324 & -13.5412 \\
\hline
\end{tabular}

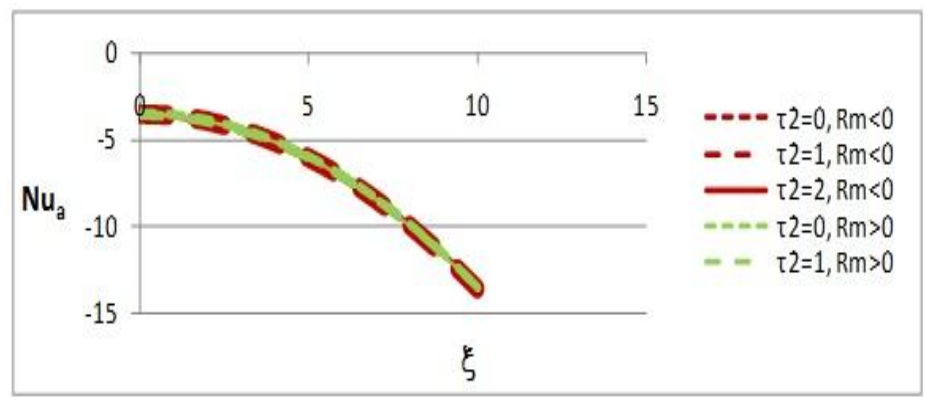

Figure-5 Variation of $N u_{a}$ with $\xi$ for different values of $\tau_{2}$ at $N=2, \xi_{0}=5$ in case of $R_{m}<0$ and $R_{m}>0$ 
Table-6 Variation of $N u_{b}$ with $\xi$ for different values of $\tau_{2}$ at $N=2, \xi_{0}=5$ in case of $R_{m}>0$ and $R_{m}<0$

\begin{tabular}{|r|c|c|c|c|c|c|}
\hline & \multicolumn{3}{|c|}{$R_{m}=-0.02$} & \multicolumn{3}{c|}{$R_{m}=0.02$} \\
\hline$\xi$ & $\tau_{2}=0$ & $\tau_{2}=1$ & $\tau_{2}=2$ & $\tau_{2}=0$ & $\tau_{2}=1$ & $\tau_{2}=2$ \\
\hline 0 & 1.555235 & 1.546435 & 1.537635 & 1.536035 & 1.544835 & 1.553635 \\
\hline 1 & 1.655235 & 1.646435 & 1.637635 & 1.636035 & 1.644835 & 1.653635 \\
\hline 2 & 1.955235 & 1.946435 & 1.937635 & 1.936035 & 1.944835 & 1.953635 \\
\hline 3 & 2.455235 & 2.446435 & 2.437635 & 2.436035 & 2.444835 & 2.453635 \\
\hline 4 & 3.155235 & 3.146435 & 3.137635 & 3.136035 & 3.144835 & 3.153635 \\
\hline 5 & 4.055235 & 4.046435 & 4.037635 & 4.036035 & 4.044835 & 4.053635 \\
\hline 6 & 5.155235 & 5.146435 & 5.137635 & 5.136035 & 5.144835 & 5.153635 \\
\hline 7 & 6.455235 & 6.446435 & 6.437635 & 6.436035 & 6.444835 & 6.453635 \\
\hline 8 & 7.955235 & 7.946434 & 7.937635 & 7.936035 & 7.944835 & 7.953635 \\
\hline 9 & 9.655234 & 9.646435 & 9.637634 & 9.636035 & 9.644835 & 9.653635 \\
\hline 10 & 11.55524 & 11.54643 & 11.53764 & 11.53604 & 11.54484 & 11.55364
\end{tabular}

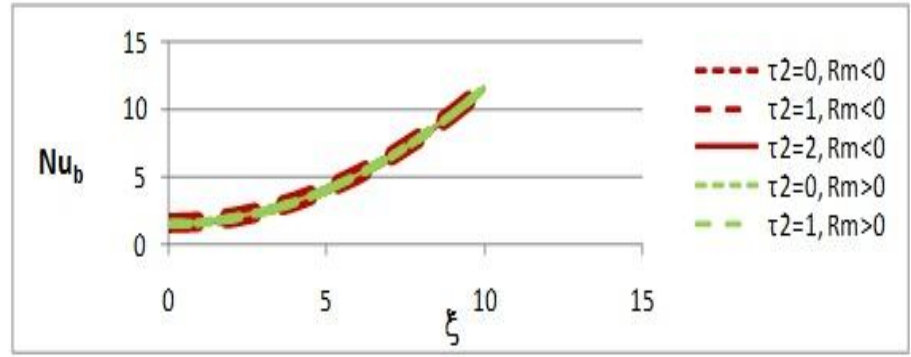

Figure-6 Variation of $N u_{b}$ with $\xi$ for different values of $\tau_{2}$ at $N=2, \xi_{0}=5$ in case of $R_{m}<0$ and $R_{m}>0$

The variation $N u_{a}$ and $N u_{b}$ with $\xi$ at $P=20, E=0.02, R_{z}=0.5, \xi_{0}=5$ for different values of $N=1,2,3$ through figure-7 and 8 respectively. The value of $N u_{a}$ is negative throughout the radial region for all values of $N$. In case of $N=1$ both $N u_{a}$ and $N u_{b}$ for net radial inflow and outflow have same values -1 for all values of $\xi$. In case of $N=2,3 N u_{a}$ have negative values whenever $N u_{b}$ have positive values. $N u_{a}$ is decreasing with an increase in velocity ratio $N$ in both cases $R_{m}<0$ and $R_{m}>0$ (see figure-7) whenever the behaviour of $N u_{b}$ (see figure-8) is just reverse to that of $N u_{a}$.

Table-7 Variation of $N u_{a}$ with $\xi$ at $\tau_{2}=2, \xi_{0}=5$ for different values of $N$ in case of $R_{m}<0$ and $R_{m}>0$

\begin{tabular}{|r|r|c|c|r|c|c|}
\hline & \multicolumn{3}{|c|}{$R_{m}=-0.02$} & \multicolumn{3}{c|}{$R_{m}=0.02$} \\
\hline$\xi$ & $N=1$ & $N=2$ & $N=3$ & $N=1$ & $N=2$ & $N=3$ \\
\hline 0 & -1 & -3.55083 & -11.2226 & -1 & -3.54123 & -11.2012 \\
\hline 1 & -1 & -3.65083 & -11.6226 & -1 & -3.64123 & -11.6012 \\
\hline 2 & -1 & -3.95083 & -12.8226 & -1 & -3.94123 & -12.8012 \\
\hline
\end{tabular}


Heat Transfer In The Steady Flow Of A Second-Order Fluid Between Two Enclosed Discs Rotating

\begin{tabular}{|r|r|r|r|r|r|r|}
\hline 3 & -1 & -4.45083 & -14.8226 & -1 & -4.44123 & -14.8012 \\
\hline 4 & -1 & -5.15083 & -17.6226 & -1 & -5.14123 & -17.6012 \\
\hline 5 & -1 & -6.05083 & -21.2226 & -1 & -6.04123 & -21.2012 \\
\hline 6 & -1 & -7.15083 & -25.6226 & -1 & -7.14123 & -25.6012 \\
\hline 7 & -1 & -8.45083 & -30.8226 & -1 & -8.44123 & -30.8012 \\
\hline 8 & -1 & -9.95083 & -36.8226 & -1 & -9.94123 & -36.8012 \\
\hline 9 & -1 & -11.6508 & -43.6226 & -1 & -11.6412 & -43.6012 \\
\hline 10 & -1 & -13.5508 & -51.2226 & -1 & -13.5412 & -51.2012 \\
\hline
\end{tabular}

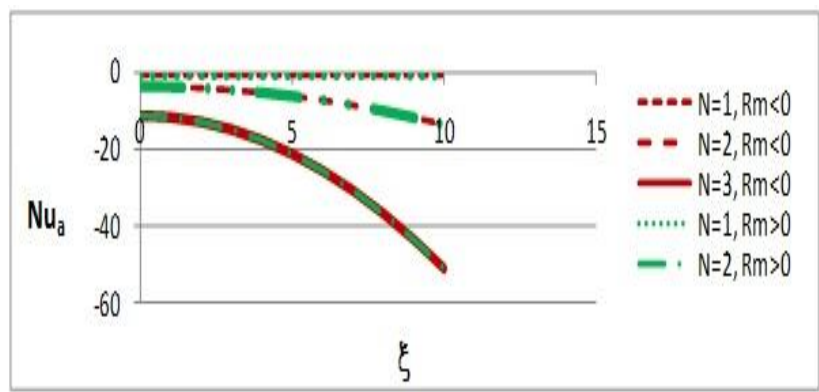

Figure-7 Variation of $N u_{a}$ with $\xi$ at $\tau_{2}=2, \xi_{0}=5$ for different values of $N$ in case of $R_{m}<0$ and $R_{m}>0$

Table-8 Variation of $N u_{b}$ with $\xi$ at $\tau_{2}=2, \xi_{0}=5$ for different values of $N$ in case of $R_{m}<0$ and $R_{m}>0$

\begin{tabular}{|r|r|r|r|r|r|r|}
\hline & \multicolumn{3}{|c|}{$R_{m}=-0.02$} & \multicolumn{3}{c|}{$R_{m}=0.02$} \\
\hline$\xi$ & $N=1$ & $N=2$ & $N=3$ & $N=1$ & $N=2$ & $N=3$ \\
\hline 0 & -1 & 1.537635 & 9.186851 & -1 & 1.553635 & 9.233784 \\
\hline 1 & -1 & 1.637635 & 9.58685 & -1 & 1.653635 & 9.633784 \\
\hline 2 & -1 & 1.937635 & 10.78685 & -1 & 1.953635 & 10.83378 \\
\hline 3 & -1 & 2.437635 & 12.78685 & -1 & 2.453635 & 12.83378 \\
\hline 4 & -1 & 3.137635 & 15.58685 & -1 & 3.153635 & 15.63378 \\
\hline 5 & -1 & 4.037635 & 19.18685 & -1 & 4.053635 & 19.23378 \\
\hline 6 & -1 & 5.137635 & 23.58685 & -1 & 5.153635 & 23.63378 \\
\hline 7 & -1 & 6.437635 & 28.78685 & -1 & 6.453635 & 28.83378 \\
\hline 8 & -1 & 7.937635 & 34.78685 & -1 & 7.953635 & 34.83378 \\
\hline 9 & -1 & 9.637634 & 41.58685 & -1 & 9.653635 & 41.63378 \\
\hline 10 & -1 & 11.53764 & 49.18685 & -1 & 11.55364 & 49.23378 \\
\hline
\end{tabular}

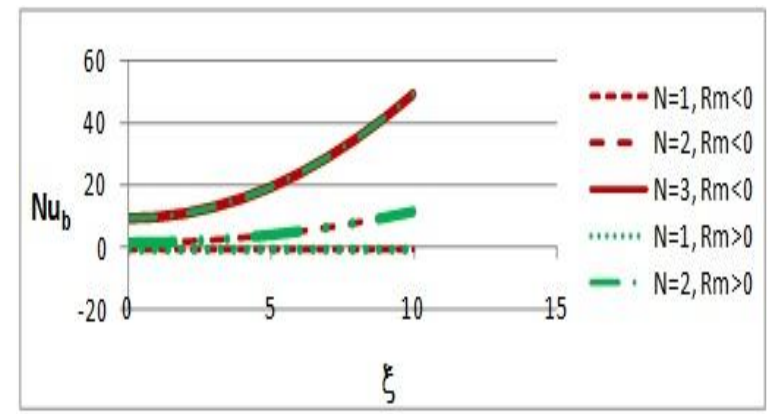

Figure-8 Variation of $N u_{b}$ with $\xi$ at $\tau_{2}=2, \xi_{0}=5$ for different values of $N$ in case of $R_{m}<0$ and $R_{m}>0$ 


\section{Conclusions}

It is evident from the expressions of the functions $H, G, L, M^{\prime}$ and the graphs of this problem that at $N=1$ the results are identical to those obtained by Sharma and $\operatorname{Singh}^{[6]}$.

Since $N u_{a}$ is negative throughout the entire radial region it follows that the heat is being transferred from the fluid to the lower disc and the amount of heat transfer decreases with an increase in $\tau_{2}$ in case of $R_{m}>0$ whenever increases in case of $R_{m}<0$. It is also concluded that due to $N u_{b}>0$ for all values of $\xi$ heat is being transferred from the fluid towards the upper disc and the amount of heat transfer is being increased with an increase in the angular velocity of the upper disc.

\section{References}

[1] Smith, R.D., Experimental cooling of radial flow turbines, A.S.M.E., paper No. 54-A-245(1945).

[2] Emmons, H.W. and S.L.Soo, Development and preliminary test of a rotating viscometer. Harvard University research report, Cambridge, Massachusetts, May 1952.

[3] Soo, S.L., R.W. Besant and Z.N. Sarafa, the nature of heat transfer from an enclosed rotating disc, Z. Angew, Math. Phys., 13 (1962) p 297.

[4] Sharma, S.K. and R.S. Agarwal, Heat transfer from an enclosed rotating disc, Appl. Set. Res. 16 (1966) p 204.

[5] Sharma, S.K., On the flow over an enclosed rotating disc, Proc. VIII Cong. On Theor and Appl., India, 8 (1963) p 77.

[6] Sharma, H.G and K.R. Singh, Heat transfer in the flow of a second-order fluid between two enclosed rotating disc, Int. J. Heat Mass Transfer, 28 (1985) p 304.

[7] Coleman, B.D. and W. Noll, Recent result in the continuum theory of visco-elastic fluids, Annals., N. Y. Acad. Sci. 89 (1961) p 672. 